\title{
Synthesis of Zinc - Bismuth Complex Oxide and Experimental Analysis of Its Magnetic and Electrical Characteristics
}

\author{
Shirui Weng, Xianyi Zhang* \\ Anhui Normal University, Wuhu, Anhui, 241000, China
}

\section{Keywords: Zinc - Bismuth Complex Oxide; Synthesis; Magnetic and Electrical Characteristics}

\begin{abstract}
The effects of reaction material ratio and feed order on the composition of the product are investigated by the reaction of zinc sulfate, bismuth nitrate and sodium hydroxide as raw materials in the ultrasonic system. The product is identified as $\mathrm{Bi}_{2} \mathrm{Zn}_{5} \mathrm{O}_{8}$ by X-ray fluorescence and chemical titration, and the product can be characterized by TEM, SEM and TG-DTA. In this paper, the basic principle and experimental method of $\mathrm{X}$ - ray diffractometer are also introduced for the analysis of the sample structure in details. Finally, we expound the principle, application range and related parameters of the physical property measurement system (PPMS) of the experimental instrument for measuring the magneto and electric properties of the Zinc - Bismuth Complex Oxide samples.
\end{abstract}

\section{Introduction}

With the rapid development of various application technologies, the preparation and performance of complex oxide crystals, which contain zinc or bismuth complex oxide, have attracted more and more attention in both academia and industry [1]. They can be applied to many aspects, such as catalysts, electrolyte materials, optoelectronic materials, electronic ceramic powder materials, high temperature superconducting materials. In addition, they can also be applied in other materials such as nuclear waste materials, absorbing materials, in which these complex oxide crystals also have a good application prospects [2][3]. Therefore, the formation mechanism of dendrites has attracted much attention for a long time, and most of them have been focused on the fractal research. From the perspective of crystal growth, the formation of dendrites is studied from the theory of crystal interface instability. In this paper, a zinc-bismuth complex oxide is synthesized by the ultrasonic technique, and its crystal growth characteristics are studied [4]. The results show that the dendrites are formed by the use of negative ion coordination polyhedrons as growth elements in the equilibrium direction, which are often used to analyze the composition of the material, crystal structure and morphology and other important information. The most simple and most common method of the current violence is mainly the use of radiation to the crystal atoms or molecules, while the crystal grain for the formation of a similar grating structure can resulte in the coherent scattering. In addition, the coherent ray is superimposed and then the square is calculated as the scattering intensity, to which the structure of the scatterer is closely related [5][6]. And the atomic and distribution in the sample are determined by the scattering intensity and angular distribution. In order to obtain the lattice distance of the sample, the angle of incidence and the wavelength should be changed [7]. According to the same method, the measurement is also the same with that of the distance between the incident and the wavelength. The most common method is the powder method, which is used as much as possible. In this paper, the computer software can be used to record the sample at each angle above the diffraction intensity of the value, which is according to the value for obtaining the diffraction map [8]. Since we can only change the incident ray of the grazing angle for the operating equipment relative comparison, and the polycrystalline samples are easier to obtain, so the method is the most widely used powder method.

\section{Preparation and Analysis of Zinc - Bismuth Complex Oxide}

\subsection{Preparation of Zinc - Bismuth Complex Oxide}

Zinc sulfate ( $\mathrm{ZnSO}_{4} \cdot 7 \mathrm{H}_{2} \mathrm{O}$, AR), bismuth nitrate $\left(\mathrm{Bi}\left(\mathrm{NO}_{3}\right)_{3} \cdot 5 \mathrm{H}_{2} \mathrm{O}\right.$, AR), concentrated nitric acid (HNO3, AR) and sodium hydroxide $(\mathrm{NaOH}, \mathrm{AR})$ are purchased from the Sinopharm Group 
Chemical Reagent Co. without the further purification before use. In this paper, the water used in the experiments is secondary distilled water [9]. The laboratory equipments used in the experiments are as follows: Ultrasonic instrument (ZK-82B type, Shanghai Instrument Factory); ZSXminiII desktop X-ray fluorescence spectrometer; Japan TEOLTEM-100SX type transmission electron microscope (accelerated voltage 180kV) and the Japanese production (SB5200, SHANGHAIBRANSON); Electric vacuum oven HitachiX-640 scanning electron microscope (test conditions: sample spray treatment, measuring voltage 20kV); Japan University of TG-DTA thermogravimetric analyzer.

The orthogonal experiment is designed according to the amount and order shown in Table 1. After reacting in a vigorous ultrasound system for $1 \mathrm{~h}$, centrifuging and dring in vacuo at $60^{\circ} \mathrm{C}$ for 1 $\mathrm{h}$, then the products can be calcined at $500^{\circ} \mathrm{C}$.

Table 1. Ratio and feeding order of $\mathrm{Bi}(\mathrm{NO} 3$ ) 3. 5H2 O : ZnSO4· 7H2 O

\begin{tabular}{|c|c|c|c|c|c|c|}
\hline \multirow[t]{2}{*}{ Index } & \multicolumn{3}{|c|}{$\begin{array}{l}\text { No. } 1 \text { sample } \\
\text { The sodium zincate is dropped into the } \\
\text { bismuth nitrate solution }\end{array}$} & \multicolumn{3}{|c|}{$\begin{array}{c}\text { No. } 2 \text { sample } \\
\text { The bismuth nitrate is dropped into } \\
\text { sodium zincate solution }\end{array}$} \\
\hline & 1.1 & 1.2 & 1.3 & 2.1 & 2.2 & 2.3 \\
\hline $\mathrm{Bi}\left(\mathrm{NO}_{3}\right)_{3} \cdot 5 \mathrm{H}_{2} \mathrm{O}(\mathrm{g})$ & 4.92 & 4.02 & 2.54 & 2.45 & 2.45 & 2.46 \\
\hline $\mathrm{ZnSO}_{4} \cdot 7 \mathrm{H}_{2} \mathrm{O}(\mathrm{g})$ & 10.62 & 9.63 & 9.63 & 4.78 & 5.88 & 9.64 \\
\hline $\begin{array}{l}\text { Reactant } \mathrm{Bi} / \mathrm{Zn} \\
\text { molar ratio }\end{array}$ & $1: 3.3$ & $1: 4.0$ & $1: 6.7$ & $1: 3 / 4$ & $1: 4.0$ & $1: 6.5$ \\
\hline
\end{tabular}

\subsection{Quantitative analysis of complex oxide composition}

Since the content of $\mathrm{Bi}$ and $\mathrm{Zn}$ in the complex oxide is in the range of $1 \%$ to $90 \%$, the coordination titration method is used in the chemical analysis. According to the acid effect curve of ethylenediamine tetraacetic acid (EDTA), as shown in Figure 1, the content of zinc bismuth in zinc bismuth composite oxide is designed by $\mathrm{Bi}^{3+}, \mathrm{Pb}^{2+}$ continuous titration experiment in bismuth and lead mixed solution [10]. Both $\mathrm{Bi}^{3+}$ and $\mathrm{Zn}^{2+}$ can form a stable 1: 1 complex with EDTA, and $\operatorname{logK}$ are 27.94 and 16.50, respectively. According to the conditions of mixed ion stepwise titration, when $\mathrm{CM} 1=\mathrm{CM} 2, \mathrm{TE}$ is $\pm 0.1 \%$ and $\Delta \mathrm{PM}$ is \pm 0.2 , which needs $\Delta \lg K_{B i Y} \geqslant 6$. And the stability constant of $\mathrm{BiY}$ and $\mathrm{ZnY}$ is different from that of 11.44. Therefore, the titration can be carried out by controlling $\mathrm{pH}$ respectively. $\mathrm{Bi}^{3+}$ is usually titrated at $\mathrm{pH} \approx 1$ while $\mathrm{Zn}^{2+}$ is titrated at $\mathrm{pH} 4$ to 6 .

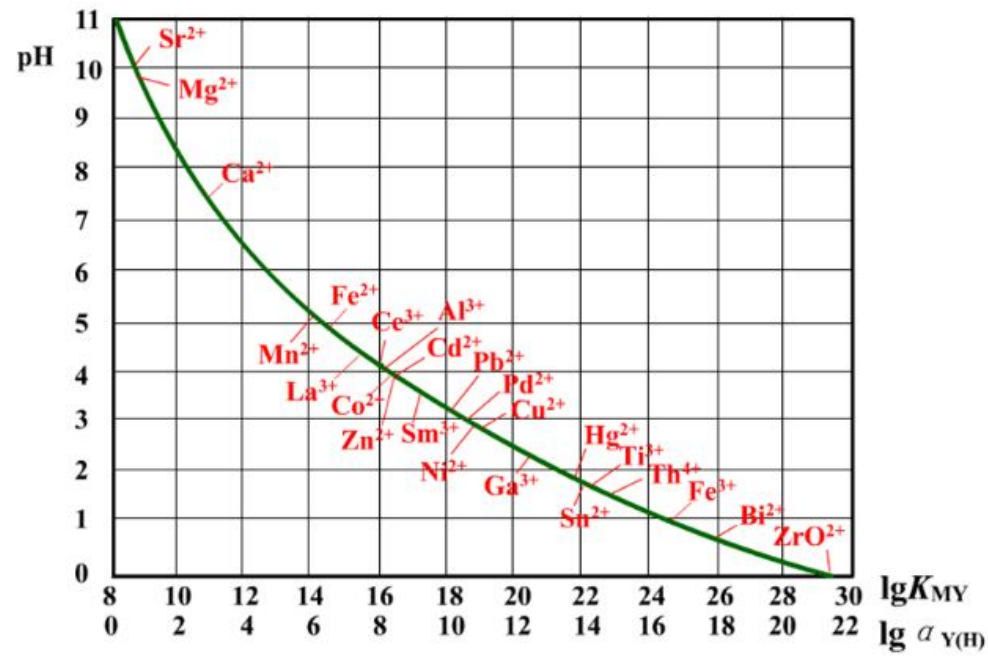

Figure 1. EDTA acid response curve

With the addition of the xylenol orange as indicator,we should continuous titrate $\mathrm{Bi}^{3+}$ and $\mathrm{Zn}^{2+}$ [11]. Once the end of the forest can meet the error formula (Equation 1) , it means that $\mathrm{Zn}^{2+}$ is without interference. 


$$
E_{t}=\frac{10^{\triangle p B i}-10^{-\Delta p B i}}{\sqrt{10^{\lg K_{B i}} \times C_{B i}^{e p}}} \times 100 \%
$$

Where $\lg K_{B i Y}^{\prime}=\lg K_{B i Y}-\lg \partial_{Y(\mathrm{Zn})}=9.44$.

According to the above principle and calculation, we can use the xylenol orange as indicator for the accurate titration of the contents of $\mathrm{Bi}$ and $\mathrm{Zn}$ with EDTA standard solution. The specific steps are: 1) Sample about $0.2 \mathrm{~g}$ with the accurately weighed, and then it is placed in $250 \mathrm{~mL}$ erlenmeyer flask. 2) Add appropriate amount of nitric acid until the sample is just completely dissolved, then add water $100 \mathrm{~mL}$ with $\mathrm{pH}$ in 1 to 2 . 3) Plus xylenol orange indicator 3 drops [12]. Titration with the EDTA standard solution, which is from purple to bright yellow, means the end of the determination of $\mathrm{Bi}^{3+}$. Then the content of $\mathrm{Bi}^{3+}$ in the sample can be calculated according to the milliliters of EDTA and the concentration of EDTA as the following equation,

$$
V\left(B i^{3+}\right)=M \times V_{1} \times 208.98 \times 100 / m \times 100
$$

Where $\mathrm{M}$ is the concentration of EDTA standard solution, $V_{1}$ is the volume of EDTA standard solution consumed by titration $\mathrm{Bi}^{3+}$, $\mathrm{m}$ is the mass of the sample.

After titration of $\mathrm{Bi}^{3+}$, we add 1 drop of the xylenol orange indicator. And then we add $25 \%$ hexamethylene tetramine into the solution to stabilize the purple, and then over $5 \mathrm{~m}$ [8]. And then the solution $\mathrm{pH}$ is about 4 to 5, then EDTA titration to the solution from purple to bright yellow is used as the end of the line. The content of $\mathrm{Zn}^{2+}$ in the sample is calculated according to the milliliters of EDTA and the concentration of EDTA as the following equation,

$$
V\left(Z n^{2+}\right)=M \times V_{2} \times 65.39 \times 100 / m \times 100
$$

Where $\mathrm{M}$ and $\mathrm{m}$ are as defined above and $V_{2}$ is the volume of EDTA standard solution consumed by titration $\mathrm{Zn}^{2+}$.

\subsection{Result analysis}

As a result of the titration, it is found that the molar ratio of the product is also increased as the molar ratio of the reactants increased when the No. 1 sample (the sodium zincate is dropped into the bismuth nitrate solution). And when the No. 2 sample (bismuth nitrate sodium zincate solution)is reacting, the molar ratio of reactants is increased, while the molar ratio of the product does not change much. And when the reaction molar ratio is between 1: 3.3 and 1: 4.0, the product ratio is 2: 5 . Therefore, we take the sample No. 2.2 as a precursor, and get the final product at $500{ }^{\circ} \mathrm{C}$ under the conditions of calcination. The results of titration are given in details as shown in Table 2.

Table 2. Results of titration

\begin{tabular}{c|c|c|c|c|c|c}
\hline Index & $\mathbf{1 . 1}$ & $\mathbf{1 . 2}$ & $\mathbf{1 . 3}$ & $\mathbf{2 . 1}$ & $\mathbf{2 . 2}$ & $\mathbf{2 . 3}$ \\
\hline $\begin{array}{c}\text { Reactant Bi / Zn } \\
\text { molar ratio }\end{array}$ & $1: 3.3$ & $1: 4.0$ & $1: 6.5$ & $1: 4.0$ & $1: 3.3$ & $1: 6.5$ \\
\hline $\begin{array}{c}\text { Product Bi / Zn } \\
\text { molar ratio }\end{array}$ & $1: 2$ & $1.5: 2$ & $13: 1$ & $2: 5$ & $2: 5$ & $1: 2.1$ \\
\hline
\end{tabular}

The results show that the molecular weight of the precursor is $\mathrm{Bi}_{2} \mathrm{O}_{3} \cdot 5 \mathrm{ZnO} \cdot \mathrm{xH}_{2} \mathrm{O}(x=6 \sim 10)$, and the content of oxygen in the product is $16.2 \%$ by $\mathrm{X}$-ray fluorescence after high temperature calcination, and the product can be deduced from $\mathrm{Bi}_{2} \mathrm{Zn}_{5} \mathrm{O}_{8}$.

\section{Magneto and electric properties measurement principle}

\subsection{Method and principle of magnetic property measurement}

Quantum Design (Quantum Design) designs and products the integrated physical properties of the measurement system (Physical Property Measurement System-PPMS), which can be a variety of physical properties of materials for high-precision testing. With the different options, we can measure the magnetic properties and electrical transport properties as we need [3][9]. In addition, the system can provide the ultra-low temperature $(1.9 \mathrm{~K})$, and the use of immersion in liquid helium in the 
superconducting excitation can produce a strong magnetic field ( \pm 9T). The use of high-speed molecular spring can make the sample cavity less than 20Toor vacuum environment, which can ensure the accuracy of the measurement results. The system integrates the MultiVu measurement software, which can be automatically measured according to the need for parameter setting [11]. The system can also purchase a variety of options to measure electrical, specific heat and other properties. The example of Physical Property Measurement System-PPMS is shown in Figure 2.

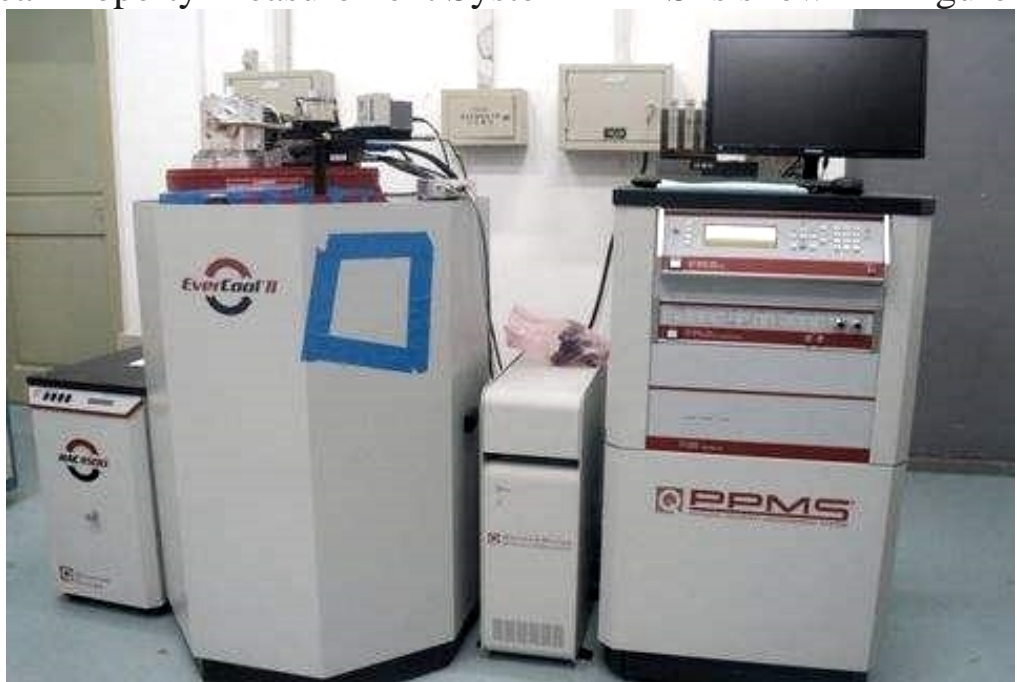

Figure 2. Example of Physical Property Measurement System-PPMS

The sample DC and AC magnetic susceptibilities are measured with using the optional ACMS for PPMS as shown in Figure 3. The AC magnetic susceptibility (real part) and DC magnetization of the sample can be obtained by measuring the DC magnetization property (ACMS). This is because the ACMS uses a unique detection coil and a drive motor. And through the coil compensation and multi-point measurement, the noise generated inside and ousider the equipment can be eliminated effectively [2][4]. At the same time, a digital thermometer in the sample cavity near the sample can be used to measure the temperature range from $1.9 \mathrm{~K}-350 \mathrm{~K}$. In order to close from the sample, it is the ACMS coil to improve the measurement accuracy.

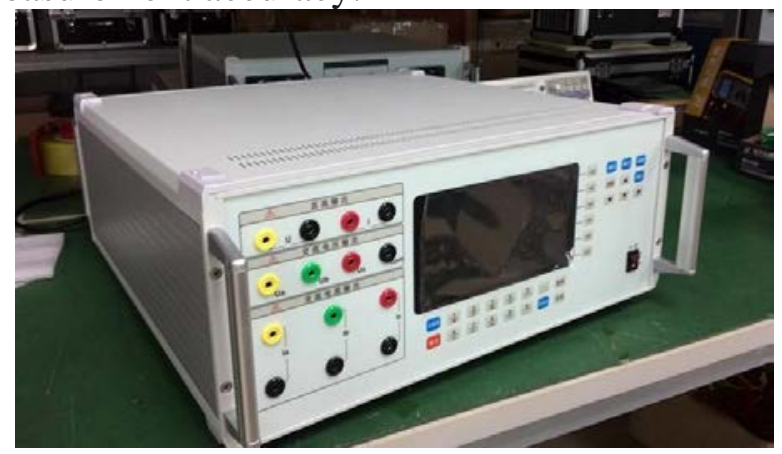

Figure 3. AC and DC magnetic properties measurement options (ACMS)

The DC magnetization measured by ACMS is all the sum of the magnetic moment at a certain temperature. And AC magnetic can susceptibility measure with using a phase amplification technology [6][9]. And the AC components can produce a small AC change the magnetic field, so as to obtain the magnetic moment response of the sample and directly measure the magnetic susceptibility. When conducting magnetic measurements, it is necessary to make the sample into a regular size of the regular cubes, and use the electronic balance to weigh. In this paper, we put it in the special sample tube with a special tape fixed on the ACMS sample tube after the observation. The sample tube and tape are specially made, which are made of a very weak magnetic signal and special materials to minimize its impact on the accuracy of the experimental results [10][11].

\subsection{Measurement method and principle of transport property}

With application of the DC transport option (measuring the nature of electrical transport), you can measure a sample at each time, and the sample DC resistivity measurement method can use a more accurate four-lead method. A measurement can get a lot of data, such as resistivity, Huo 
Coefficient and other series of data, while the resistivity of the sample with the shape of the irregular but uniform thickness can be measured by the method.

The electrical transport properties of the sample mainly refer to the relationship between the resistivity and temperature. In this paper, we measure the resistivity of the current method commonly used in the four-lead method. First of all, the first sample should be drought on the four copper wire as a diversion probe (as shown in Figure 4). And then we use the silver paint to connect the probe with the sample, so that the probe can be evenly attached to the measured sample with a constant current source from the outermost two current source access current. Then we measure the middle two voltage of the probes, so the resistance value of the sample can be calculated from the voltage and the current, thereby we can obtain the resistivity [2].

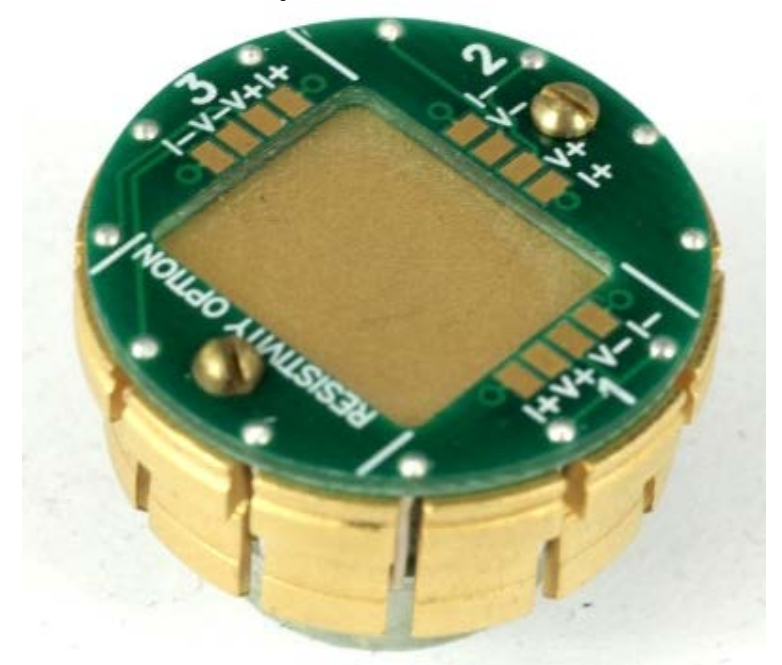

Figure 4. The sample tray used to measure the resistance with PPMS

The four-lead method can significantly reduce the thermoelectric power of the probe and other factors as compared with the traditional two-lead method. Especially, a small measured resistance value of the sample also include the probe resistance and contact resistance. And the four-lead method can make the current through the voltmete [8]. In addition, it has a higher degree of accuracy and can be degrees with the actual voltage and current of the sample itself. After setting the program, the two channels can measure the voltage and the resistance value simultaneously according to the change of the outside temperature and the magnetic field [12]. Due to the size of the sample holder, the size of the sample should be measured and it needs to meet the requirement, and the shape should be regular and uniform in thickness. Before measuring, we use the vernier caliper to measure the actual size of the sample and the distance between the contacts and enter the system. And at the end of each measurement, the measurement software can automatically calculate the resistivity of the sample based on the input size and the contact pitch to facilitate the subsequent analysis and calculation.

\section{Summary}

This paper mainly introduces the preparation methods of synthetic Zinc-Bismuth oxide, and elaborates the principle of preparing the series of samples by the high temperature solid state reaction method. And the basic principle and experimental method of X-ray diffractometer are also introduced in details for the sample structure analysis. Finally, the principle, application range and related parameters of the physical properties measurement system (PPMS) are measured to improve the accuracy of the measurement of the magnetic and electrical characteristics. With using PPMS to measure the magnetic properties of the sample, the maximum magnetic field can reach $\pm 9 \mathrm{~T}$, and the minimum temperature can reach $1.9 \mathrm{~K}$. In this paper, the method of measuring the resistivity is connected by a four-lead method to the samples selected by the PPMS to measure the electrical transport properties, and the measurement results can guarantee the extremely high accuracy. 


\section{Acknowledgements}

This work was supported by National Natural Science Foundation of China (31401285 、 61475163).

\section{References}

[1] Gutknecht T, Gustafsson A, Forsgren C, et al. Investigations into recycling zinc from used metal oxide varistors via $\mathrm{pH}$ selective leaching: characterization, leaching, and residue analysis[J]. The Scientific World Journal, 2015, 2015.

[2] Jalkanen P, Tuboltsev V, Marchand B, et al. Magnetic properties of polycrystalline bismuth ferrite thin films grown by atomic layer deposition[J]. The journal of physical chemistry letters, 2014, 5(24): 4319-4323.

[3] McCloy J S, Jiang W, Bennett W, et al. Electrical and Magnetic Properties Modification in Heavy Ion Irradiated Nanograin Ni x Co (3-x) O4 Films[J]. The Journal of Physical Chemistry C, 2015, 119(39): 22465-22476.

[4] Chon M P, Tan K B, Zainal Z, et al. Synthesis and Electrical Properties of Zn - substituted Bismuth Copper Tantalate Pyrochlores[J]. International Journal of Applied Ceramic Technology, 2016, 13(4): 718-725.

[5] Marchand B, Jalkanen P, Tuboltsev V, et al. Electric and Magnetic Properties of ALD-Grown BiFeO3 Films[J]. The Journal of Physical Chemistry C, 2016, 120(13): 7313-7322.

[6] Mei L T, Hsiang H I, Hsu W H. Varistor and Magnetic Properties of Nickel Copper Zinc Niobium Ferrite Doped with Bi2O3[J]. Journal of the American Ceramic Society, 2014, 97(12): 3918-3925.

[7] Gutiérrez - López J, Masó N, Levenfeld B, et al. Electrical and Magnetic Properties of NiZn Ferrite Prepared by Conventional and Solar Sintering[J]. Journal of the American Ceramic Society, 2016, 99(7): 2327-2333.

[8] Kumar N, Shukla A, Behera C, et al. Structural, electrical and magnetic properties of Bi (Ni 0.45 Ti 0.45 Fe 0.10) O 3[J]. Journal of Alloys and Compounds, 2016, 688: 858-869.

[9] Egorysheva A V, Ellert O G, Zubavichus Y V, et al. New complex bismuth oxides in the Bi 2 O 3-NiO-Sb 2 O 5 system and their properties[J]. Journal of Solid State Chemistry, 2015, 225: 97-104.

[10] Medeiros P N, Gomes Y F, Bomio M R D, et al. Influence of variables on the synthesis of CoFe2O4 pigment by the complex polymerization method[J]. Journal of Advanced Ceramics, 2015, 4(2): 135-141.

[11] Rout J, Choudhary R N P, Sharma H B, et al. Effect of co-substitutions (Ca-Mn) on structural, electrical and magnetic characteristics of bismuth ferrite[J]. Ceramics International, 2015, 41(7): 9078-9087.

[12] Khalid M, Rabab S. Influence Of Zinc Oxide Particle Size And Surface Properties On The Electrical, Optical And Cytotoxicity Characteristics Of Zinc Oxide Discs[D]. Universiti Sains Malaysia, 2015. 